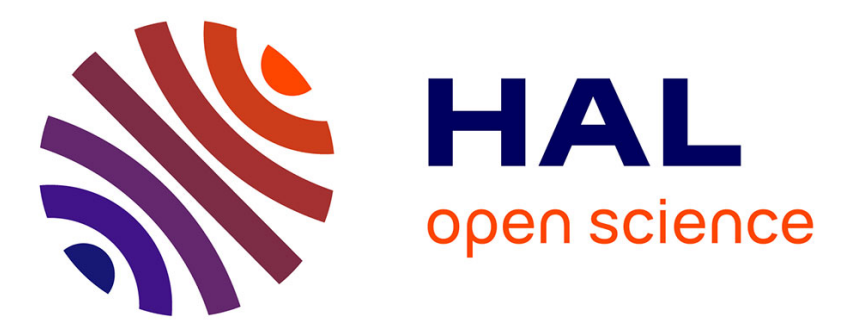

\title{
Apport du laboratoire pour le diagnostic des myiases
}

Claude Guiguen, Sorya Belaz, Dominique Chabasse, Jean-Claude Beaucournu

\section{To cite this version:}

Claude Guiguen, Sorya Belaz, Dominique Chabasse, Jean-Claude Beaucournu. Apport du laboratoire pour le diagnostic des myiases. Revue Francophone des Laboratoires, 2020, 2020 (522), pp.72-80. 10.1016/S1773-035X(20)30167-2 . hal-02948494

\section{HAL Id: hal-02948494 \\ https://hal-univ-rennes1.archives-ouvertes.fr/hal-02948494}

Submitted on 25 Sep 2020

HAL is a multi-disciplinary open access archive for the deposit and dissemination of scientific research documents, whether they are published or not. The documents may come from teaching and research institutions in France or abroad, or from public or private research centers.
L'archive ouverte pluridisciplinaire HAL, est destinée au dépôt et à la diffusion de documents scientifiques de niveau recherche, publiés ou non, émanant des établissements d'enseignement et de recherche français ou étrangers, des laboratoires publics ou privés. 


\section{Apport du laboratoire pour le diagnostic des Myiases}

Claude Guiguen ${ }^{1}$, Sorya Belaz ${ }^{2}$, Dominique Chabasse ${ }^{3}$ et Jean-Claude Beaucournu ${ }^{1}$

1- Service de Parasitologie et Zoologie appliquée, UFR des Sciences Médicales 35043 Rennes cedex

2-Service de parasitologie - mycologie, CHU Pontchaillou, Rue Henri le Guillou, 35033 Rennes cedex

3- Pôle Santé de l'Université d'Angers, 49100 Angers

Correspondance : guiguenclaude@orange.fr

\section{Résumé}

Les myiases sont liées au parasitisme d'un être vivant par des larves de mouches ou asticots. Certaines de ces larves sont des parasites obligatoires ; d'autres vivent normalement sur des matières organiques en décomposition et se développent accidentellement chez l'homme. Sur le plan clinique on distingue les myiases cutanées, sous cutanées, cavitaires et profondes. Une clé sommaire d'identification des asticots responsables des myiases les plus fréquentes (uniquement sur les stades III) est proposée tout en sachant que cette identification est affaire de spécialiste. Le traitement se résume à l'extraction manuelle des larves et au décapage minutieux des plaies. Dans I'hypodermose I'ivermectine est active.

\section{Mots clefs}

Asticot, myiase accidentelle, myiase cutanée, myiase obligatoire

\section{Summary}

\section{Medical laboratory contribution to myiasis diagnosis}

Myiasis are a living being parasitism with a fly larvae or maggot. Some are obligate parasites, other live in decaying organic matters and accidentally occur on human. Four clinical types can be recognized, cutaneous, subcutaneous, luminal and deep myiasis. A brief identification key for stage III maggots responsible for myiasis is provided, though identification is a specialist matter. Treatment lay on manual removal of fly larvae and wounds careful cleansing. Furthermore, ivermectine is efficient on hypodermosis. 


\section{Key words}

Maggot, opportunist myiasis, cutaneous myasis, compulsory myiasis

\section{Introduction}

Les myiases désignent tout parasitisme d'un être vivant par les larves acéphales et apodes (asticots) de diptères cyclorraphes ou mouches. Ces larves se nourrissent, au moins pendant une période de leur vie, de tissus vivants ou morts de leur hôte ou des liquides organiques de celui-ci [1].

\section{1 - Epidémiologie}

Le cycle évolutif classique de ces insectes se déroule comme suit. Après accouplement, les femelles pondent leurs œufs (espèces ovipares) ou leurs larves (espèces larvipares) sur un substrat favorable à leur développement (sol, eau souvent putride, tige, bouton floral, fruits ou légumes,...), soit isolément, soit en trainées ou dispersés sur une surface plus ou moins grande. Certaines espèces (Calliphora) peuvent émettre soit des œufs soit des larves en fonction de la température extérieure. A maturation, les œufs libèrent un asticot de 1 à quelques $\mathrm{mm}$ de long. Le cycle comprend 3 stades larvaires. Après plusieurs mues l'asticot peut donc atteindre $2 \mathrm{~cm}$ ! À l'extrémité postérieure de celui-ci existe une paire de stigmates respiratoires dont la morphologie varie en fonction des genres et des espèces. Après un laps de temps souvent fonction de la température ambiante, la cuticule de l'asticot durcit : c'est la pupe, en tonnelet, à l'intérieur de laquelle l'insecte adulte (ou imago) se forme (figure 1). À maturité, la mouche s'échappe par un opercule préformé d'où le terme de cyclorraphe. Le mâle et la femelle s'accouplent et le cycle recommence [2].

Les mouches se retrouvent dans toutes les contrées du monde (sauf arctique et antarctiques). Certains genres sont presque cosmopolites (Calliphora, Lucilia, Hypoderma...) d'autres localisées à une région du globe (Cordylobia en Afrique noire; Dermatobia en Amérique du Sud). Les myiases humaines sont provoquées, pour la plupart, par des espèces opportunistes se développant dans des tissus lésés ou dans des orifices naturels, mais certaines sont des parasites obligatoires. Les asticots peuvent rester en surface de la peau (myiases épicutanées hématophages : ver de case), déterger des plaies (larvothérapie par Lucilia spp.), pénétrer des cavités (Oestrus ovis dans le nez, Musca domestica dans le vagin), se fixer temporairement sur la cornée ou la conjonctive (O. ovis), pénétrer la peau (myiases sous-cutanées soit furonculeuses (ver de Cayor, ver macaque), soit 
rampantes (Gasterophilus)), ou effectuer un cycle interne tissulaire (Hypoderma, en impasse parasitaire chez l'homme) [1, 2].

\section{2 - Physiopathologie}

Les larves pénètrent et se développent dans les cavités ou dans les tissus. Elles sont à l'origine de lésions prurigineuses, inflammatoires délabrantes selon le type de myiases. Dans l'hypodermose, la migration tissulaire prolongée de la larve entraîne des réactions œdémateuses méningoencéphalitiques et/ou oculaires, accompagnée d'une hyper éosinophilie majeure [3].

\section{3 - Signes cliniques d'orientation}

Une importante contribution à l'étude des myiases a été publiée par Zumpt [4]. Les formes cliniques et la distribution géographique des différentes espèces de mouches myiasigènes figurent dans le Tableau I [2,5].

\section{1- Myiases opportunistes ou accidentelles}

Les larves de très nombreuses mouches, vivant normalement dans des matières organiques en voie de putréfaction ou non, peuvent se rencontrer chez l'homme. Elles sont localisées dans les cavités naturelles (oreille, sinus, rectum, vagin,...), dans les plis cutanés (sillon sous mammaire, plis inguinaux,...) et sont donc temporaires provoquant un prurit local. Certains asticots peuvent également se rencontrer aussi bien sur des cadavres que sur des plaies d'animaux ou d'hommes, blessés ou comateux (accidents de la voie publique, armes à feu, armes blanches, ulcères variqueux,...) voire de grabataires. Les genres classiques en Europe sont les suivants : les asticots de Lucilia spp. (mouche verte de la viande), Calliphora erythrocephala (mouche bleue de la viande), Musca domestica (mouche domestique), Eristalis tenax (larve à queue de rat), Fannia spp. fréquents sur les substances alimentaires avariées (viande, par exemple) ou les eaux saturées de fèces. Les myiases vaginales se rencontrent chez des femmes à hygiène intime rudimentaire et sont dues le plus souvent à Musca domestica. Les myiases du tube digestif dues aux larves d'Eristalis et de Fannia entrainent chez le patient des douleurs intestinales diffuses accompagnées d'épisodes diarrhéiques et de sensation de gêne anale. Ce parasitisme de l'ampoule rectale voire du bas colon résulte du fait que, en ce qui concerne les Eristalis, les adultes viennent pondre sur la marge anale et que par 
" rétro invasion » les asticots remontent dans le rectum, respirant l'air extérieur grâce à un long siphon d'où le nom « vers à queue de rat » (figure2) [1].

Il convient également de rappeler l'emploi pendant la guerre de 1914-1918 d'une Lucilia (L. sericata) dont les asticots, élevés aseptiquement, étaient déposés sur les plaies des blessés ou lors d'ostéomyélite chronique. En supprimant sélectivement les chairs mortes, les asticots nettoyaient la blessure et accéléraient la guérison. Cette méthode redevient d'actualité (larvothérapie ou magothérapie) [6,7,8]. Enfin, les asticots font partie des différentes escouades d'arthropodes retrouvés sur les cadavres en milieu naturel et donc peuvent être utilisés par la police scientifique pour essayer de dater la mort (9).

\section{2- Myiases obligatoires}

Plusieurs entités cliniques peuvent se rencontrer $[5,10]$ :

Myiases épicutanées dues à des larves ectoparasites hématophages ou vers de case, vivant aux environs des enclos à bétail, des niches de chien, des terriers de mammifères mais aussi des maisons et des cases. Dans les sols meubles de celles-ci, ou se cachant sous les nattes ou dans les interstices de plancher dans la journée elles viennent, la nuit, se gorger de sang sur les mammifères, dont l'homme, dormant à même le sol. Sa morsure peut être désagréable mais passe souvent inaperçue, semble sans gravité et n'a aucun rôle vecteur. Cette mouche d'Afrique noire se nomme Auchmeromyia senegalensis (11).

Myiases furonculeuses dues à Cordylobia anthropophaga (ver de Cayor), mouche péri domestique d'Afrique noire et à Dermatobia hominis (ver macaque), mouche selvatique d'Amérique latine. Les asticots se développent directement sous le derme au niveau de leur lieu de pénétration donnant des papules inflammatoires ayant un aspect de furoncle douloureux. On peut observer 2 petits points noirs au centre de ce pseudo furoncle correspondant aux 2 plaques stigmatiques de respiration situées à la partie postérieure de la larve (figure 3). Ces myiases furonculeuses se rencontre de plus en plus en France métropolitaine $[12,13]$ chez les voyageurs en provenance d'Afrique ou d'Amérique du Sud $[14,15]$. 
Pour le "ver de Cayor» la spécificité est très large, mais essentiellement dans l'entourage de l'homme; en dehors de celui-ci, rats, chiens, porcs... sont également très parasités [16]. La contamination se fait par contact direct avec les œufs en enfilant des vêtements imbibés de sueur, mis à sécher au soleil et non repassés ou en s'asseyant sur un sol souillé par des urines voire des excrétas [17]. II n'y a pas de cycle migratoire dans l'organisme. La larve de stade 1 s'enfonce là où elle est déposée, donne une papule inflammatoire " furonculeuse " qui évolue très rapidement (10 à 15 jours environ) avec une sensation de corps étranger mobile. Le parasitisme, pouvant être important en nombre de larves, est sans gravité (figure 4) [18].Une autre espèce Cordylobia rodhaini, non synanthrope, peut parfois parasiter I'homme ayant séjourné en zone forestière [19].

Pour le «ver macaque» ou Torcel, l'évolution est très voisine de celle de Cordylobia mais beaucoup plus lente (de 1 à 6 mois). Le parasitisme de l'homme est plus rare car la plupart des hôtes sont selvatiques. L'aspect très particulier de la larve au stade 2, en « courge " ou en " poire ", et le grand développement de ses épines cuticulaires rendent difficile et douloureuse leur extraction par simple pression (figure 5) [20]. II faut noter le comportement de ponte particulier de cette espèce qui colle ses œufs en " bande " sur un autre arthropode zoophile ou anthropophile. Lors du repas de ce dernier bardé comme un kamikaze les œufs éclosent sous l'influence de la chaleur de l'hôte. Il s'agit donc d'une véritable phorésie [21].

Myiases sous cutanées rampantes dues à Gasterophilus intestinalis ou G. inermis dont les larves chez ses hôtes classiques, les équidés, sont des parasites du tube digestif en fin de cycle. Les œufs sont collés en vol sur les poils et après ingestion par léchage ou pénétration transcutanée, les larves se fixent sur la paroi intestinale du cheval. Chez I'homme elles provoquent des sillons cutanés au tracé capricieux, érythémateux et prurigineux, type "larva migrans ». Ces affections sont rarissimes chez ce dernier et se rencontre chez les personnes vivant proche d'équidés. Elles guérissent spontanément dans des délais de quelques jours à plusieurs semaines [22].

Myiases des plaies dues aux asticots d'une espèce américaine : Cochliomyia hominivorax (lucilie bouchère) [23], d'une espèce africaine et asiatique : Chrysomia bezziana [24] et d'une espèce européenne :Wohlfahrtia magnifica (mouche des alpages) [25,26]. Ces mouches pondent leurs œufs à la surface des plaies dues à un traumatisme quelconque mais 
aussi dans les fosses nasales, l'œil et les oreilles des animaux ou de l'homme. Ces larves, peuvent envahir les tissus et entrainer des lésions spectaculaires avec délabrements considérables voire la mort (figure 6). Heureusement ces myiases restent rares chez l'homme. Le parasitisme par Wohlfahrtia est, de loin, le plus grave de toutes les myiases métropolitaines.

$>\quad$ Myiases cavitaires dues à des mouches larvipares qui déposent, en vol, leurs asticots dans les nasaux des ovins, des caprins et des équins. Les larves gagent les sinus grâce à leurs puissants crochets buccaux ou ils atteignent leur plein développement en 4 semaines. Elles se décrochent et sont rejetées au sol lorsque l'animal "éternue " pour évacuer ces corps étrangers. Après s'être enfoncées superficiellement dans le sol, elles nymphosent puis donnent un adulte. Chez l'homme cela se traduit par des rhinorrhées plus ou moins purulentes accompagnées de céphalées frontales. Cette "sinusite» connaît son étiologie lors de la sortie d'une larve au cours d'un éternuement ou d'un mouchage) [27]. Ces mouches peuvent également projeter leur ponte sur la conjonctive oculaire, ce qui est ressenti comme un simple choc au niveau de l'œil. II s'ensuit une intense irritation oculopalpébrale: sensation de brûlure, de picotements et de corps étrangers (ophtalmo myiase externe) (figure 7). L'examen de l'œil décèle une ou plusieurs larves translucides et minuscules accrochées aux muqueuses des plis palpébraux et au globe oculaire [28]. Les mouches responsables Oestrus ovis et Rhinoestrus purpureus se rencontrent essentiellement sur le pourtour du bassin méditerranéen et en Afrique noire.

$>\quad$ Myiases profondes dues à Hypoderma bovis, mouche inféodée aux bovins domestique (Bos taurus) et tout particulièrement connue des vétérinaires. L'imago se rencontre en été dans les pâturages fréquentés par le bétail dans les régions tempérées de I'hémisphère nord. La femelle colle ses œufs isolément, pendant les jours les plus chauds, sur les poils des animaux. Dès l'éclosion la larve pénètre directement au travers de la peau à la base du poil et migre dans la lumière du canal rachidien. Elle y passera tout I'hiver. Au printemps et après avoir muée, elle gagne la région dorsolombaire et vient se positionner sous la peau formant un nodule sous cutané de la taille d'une petite mandarine appelé varron. L'asticot de stade III ( $2 \mathrm{~cm}$ de long) perfore la peau. C'est par ce pertuis que la larve se laisse tomber au sol, s'y enfonce et se transforme en nymphe avant de devenir adulte. La parasitose se traduit par une diminution de la production de viande et de lait et par l'impossibilité 
d'utiliser la peau dans l'industrie du cuir [29]. Cette parasitose bovine est désormais sous contrôle en France [30]. Chez l'homme, le cycle est toujours abortif ; la larve ne dépasse pas le stade 1 voire 2. En dehors de la sortie possible par la peau au niveau d'une lésion furonculeuse, trois manifestations cliniques sont décrites:

- oedèmes pseudo tumoraux prurigineux, fugaces et mobiles apparaissant au niveau du tronc et de la tête (figure 8);

- signes neurologiques se manifestant par un syndrome méningé d'apparition brutale avec une importante éosinophilie du LCR (méningite à éosinophiles) ;

- localisations oculaires possibles et de pronostic souvent grave, l'asticot, au cours de sa migration, pouvant pénétrer dans les chambres de l'œil (ophtalmo myiase interne) (figure.9).

Cette affection, devenue très rare, touche exclusivement les enfants d'origine rurale ou ayant séjourné à la campagne pendant les vacances d'été, période de ponte des insectes. La période d'apparition des troubles s'étale d'octobre à mars avec un maximum net en janvier [31].

\section{4 - Diagnose}

\section{1- Diagnostic clinique}

Le diagnostic de " myiase " au sens large est évident si un ou des asticots sont visibles au fond d'un pertuis, ou sont apportés par le patient. Dans ce dernier cas toutefois, il faut pousser l'interrogatoire pour éviter de tomber dans le piège d'un syndrome d'Ekbom. Dans I'hypodermose une hyper éosinophilie sanguine et/ou méningée majeure peut orienter le diagnostic mais le diagnostic de certitude repose sur la sérologie (antigène extrait de la larve de stade I d'Hypoderma bovis) et rarement sur l'identification de la larve [32]. En cas de positivité, une surveillance régulière $d u$ fond d'œil doit être instituée. On évoquera une myiase rampante à Gasterophilus devant un sillon migrateur situé dans le cou ou sur le haut du thorax chez un patient s'occupant de chevaux ou vivant à la campagne près de station d'élevage d'équidés et n'ayant pas voyagé en zone tropicale (élimination d'un larbish (ankylostome) et d'une larva currens (anguillule). Dans les autres myiases le diagnostic est porté par la visualisation des asticots. Ceux-ci seront prélevés à la pince ou à la curette mousse (figure 10) (myiases conjonctivales, des plaies, des plis cutanés, des cavités naturelles, 
...) ou après exérèse pour les myiases furonculeuses voire par leur expulsion lors d'un éternuement ou d'un mouchage pour les myiases nasales et sinusiennes ou dans les selles pour les myiases digestives ou rectales (Erystalis tenax, Fannia spp [33]). Ces dernières peuvent parfois être vues lors d'une rectoscopie ou d'une colonoscopie basse et extirpées au cours de l'examen.

\section{2- Diagnostic entomologique}

Les asticots recueillis doivent être fixés à l'alcool éthylique à $70^{\circ}$. L'identification de l'espèce en cause nécessite, sauf cas particulier, l'aide d'un entomologiste averti. Celle-ci est réalisée sur la larve de stade III (sauf pour Hypoderma bovis où c'est le stade I). La taille, la forme, la couleur, les ornementations participent à cette identification. Mais c'est essentiellement en observant l'extrémité postérieure ou se situent les stigmates respiratoires (ou spiracles) dont la morphologie varie en fonction des genres et des espèces que celle-ci sera effectuée avec certitude. Un spiracle est constitué d'un péritrème et d'une structure circulaire ou « bouton » sclérotisés entourant (sauf chez les Oestridae et les Hypodermatidae) les fentes respiratoires (une au stade I, deux au stade II et trois au stade III, c'est ce stade le plus caractéristique). Elle repose également sur la forme des sclérites buccaux (crochets) situés à l'extrémité antérieure. L'identification nécessite donc, après dissection et déshydratation, de monter les extrémités des larves entre lame et lamelle, dans du Baume du Canada. Une clé de détermination à partir des formes cliniques est proposée dans le tableau 2 [34].

\section{5 - Traitement et prophylaxie}

II n'existe pas de traitement pour les myiases rampantes à Gasterophilus. Par contre, la présence d'asticots sur une plaie, dans les plis cutanés, dans une cavité naturelle (vagin, conduit auditif, narine,...), ou sous la conjonctive de l'œil impose leur extraction à la pince ou à la curette mousse, accompagnée parfois d'un parage chirurgical.

Si le malade présente un ou plusieurs pseudo furoncles et revient d'Afrique depuis moins de 1 mois ou d'Amérique depuis moins de 6 ou 7 mois, deux attitudes sont possibles :

- une active, qui extrait l'asticot chirurgicalement ou par pression des doigts (figure 11). 
- une passive, qui laisse le parasite s'expulser seul, ou qui consiste à recouvrir le furoncle de plusieurs épaisseurs de tulle gras ou de pommade antibiotique avec surveillance quotidienne ; la larve cherchant à respirer se retrouve dans le tulle ou la pommade.

Dans tous les cas, il convient d'éviter la surinfection en appliquant un désinfectant et un pansement léger et de vérifier la vaccination antitétanique [35].

L'utilisation systématique de l'ivermectine en France a entrainé des résultats spectaculaires dans l'hypodermose bovine et de ce fait engendré une diminution des cas humains. Cette même molécule utilisée à la dose de $200 \mu \mathrm{g} / \mathrm{kg}$ en prise unique, à jeun, et sans manger pendant les 2 heures suivantes, a donné de bons résultats dans l'hypodermose humaine. Cependant elle est contre-indiquée dans les localisations oculaires où le traitement reste chirurgical. A noter que l'ivermectine diluée à $10 \%$ dans du propylène- glycol semble une alternative intéressante dans la prise en charge des myiases dues à $C$. hominivorax. Son emploie entraine l'immobilisation des larves et parallèlement une sédation des douleurs [36]. Des corticoïdes locaux peuvent être administrés s'il y a inflammation.

La prophylaxie des myiases est individuelle (hygiène corporelle; pansement aseptique des plaies; repassage avec un fer chaud ou séchage du linge à l'abri des mouches (C. anthropophaga) et collective (lutte chimique contre les mouches mais la fréquence des résistances aux insecticides habituels la rende difficile. La lutte biologique par stérilisation des mâles a été entreprise avec succès pour l'espèce Cochlyomyia hominivorax aussi bien sur le sol américain [37] qu'en Libye après son introduction en 1988 [38] ; cela nécessite une surveillance permanente des troupeaux [39]. La lutte génétique par translocation chromosomique a également été utilisée contre Lucilia cuprinata en Australie [40].

\section{Conclusion}

Le diagnostic de " myiase » est relativement aisé pour le clinicien mais cela se complique lorsque l'on veut désigner précisément l'espère parasitaire en cause. L'origine géographique ou le pays de séjour pour les voyageurs associé à la clinique est une aide précieuse mais le biologiste posera le diagnostic d'espèce sur la structure typique des trachées des larves de troisième stade voire sur les crochets buccaux. II doit avoir recours, au moindre doute, à un entomologiste reconnu. 
Liens d'intérêts : les auteurs déclarent ne pas avoir de liens d'intérêts.

\section{Points à retenir}

- Les myiases sont provoquées par le contact ou la pénétration des larves (asticots) de mouches (diptères cyclorraphes) chez l'homme ou l'animal.

- Les myiases obligatoires sont responsables de formes furonculeuses sous les tropiques (Dermatobia hominis, Cordylobia anthropophaga) et viscérales (Hypoderma bovis).

- Les myiases humaines accidentelles concernent les cavités naturelles: myiases auriculaires, rhino-oculaires (Oestrus ovis, Rhinoestrus purpureus), génitales (Musca domestica), rectales (Eristalis tenax), les plis cutanés et les plaies (Lucilia, Calliphora, Wohlfahrtia, Cochlyomyia).

- Le diagnostic est clinique avec identification de la larve dans les myiases furonculeuses et accidentelles. II repose sur l'hyper éosinophilie et la sérologie pour l’hypodermose.

- La thérapeutique est fondée essentiellement sur l'exérèse des asticots et parfois sur l'usage de l'ivermectine.

\section{Références}

[1] Duvalet $G$, Jacquiet $P$. Les diptères myiasigènes (Diptera) in Entomologie médicale et vétérinaire. Marseille : Ed Quae et IRD ; 2017. p 425-37.

[2] Anofel. Myiases et tungoses. In Parasitoses et mycoses des régions tropicales et tempérées. Paris: Elsevier Masson; 2016. p 261-8.

[3] Tabouret G, Lacroux C, Andreoletti O, et al. Cellular and humoral local immune responses in sheep expermentally infected with Oestrus ovis (Diptera:Oestridae). Vet Res 2003, 34: 23141.

[4] Zumpt F. Myiasis in man and animals in the old world. London: Butterworths Ed.; 1965. 267pp.

[5] Catts EP, Mullen GR. Myiasis (Muscoidea, Oestroidea) in Médicale and Veterinary Entomology. USA: Academic Press; 2002. p 317-48. 
[6] Shermann RA. Asticothérapie versus détertion conventionnelle dans le traitement des escarres. Revue Francophone de Gériatrie 2007;14:64.

[7] Wolff $\mathrm{H}$, Hansson C. Larva therapy, an effective method of ulcer debridement. Clin Exp Dermatol 2003;28:134-7.

[8]Raposio E, Bortolini S, Maistrello I, Grasso DA. Larval therapy for chronic cutaneous ulcers historical review and future perpertive. Wounds 2017; 29: 367-73.

[9] Talebzadeh F, Ghadipasha M, Gharedaghi J, et al. Insect fauna of human cadavers Theran distict. J Arthopod Borne Dis 2017; 11:363-70.

[10] Guiguen C, Gangneux JP et Beaucournu JC. Myiases humaines in Parasitoses et mycoses courantes de la peau et des phanères. Guide MédiBIO. Paris: Elsevier; 2003: p33-54.

[11] Noireau F. Infestation by Auchmeromyia senegalensis as a consequence of the adoption of non -nomadic life by Pygmies in the Congo Republic. Trans R Sos Trop Med Hyg 1992; 86: 329.

[12] Dehecq E, Nzungu PN, Cailliez JC et al. Cordylobia anthropophaga (Diptera: Calliphoridae) outside Africa a case of furuncular myiasis in a child returning from Congo. J Med Entomol 2005; 42:187-92.

[13] Poirier P, Foulet F, Bonoua C, et al. Myiase à Cordylobia anthropophaga d'origine sud africaine. Med Trop $2008 ; 68: 102-3$.

[14] Lachish T, Marhoom E, Mumcuoglu KY, et al. Myiasis in Travelers. J Travel Med 2015; 22: 232-6.

[15 ] Kuscu F, Ozsoy KM, Ulu A, et al. Furuncular Myiasis caused by Dermatobia hominis in a traveler returning from the amazon jungle ; Turkiye Parasitol Derg 2017 ; 41 : 173-6.

[16] Devienne P, Bodard P, Pinhas C. Le ver de Cayor, agent d'une myiase furonculeuse. Insectes 2004;135: 4.

[17] Kouam MK, Meutchieye F, Miegoue E, et al. Prévalence and husbandry related risk factors of myiasis in domestic cavies in the western highlands of Cameron. Epidemiol Infect 2017; 45: 339-46. 
[18] Veraldi S, Brusasco A, Süss L. Cutaneous myiasis caused by larvae of Cordylobia anthropophaga (Blanchard). Int J Dermatol 1993; 32: 184-7.

[19] Veraldi S, Serini SM, Süss L. Three cases of cutaneous myiasis caused by Cordylobia rodhaini. J Infect Dev Ctries 2014; 8: 249-51.

[20] Clyti E, Pages F, Pradinaud R. Le point sur Dermatobia hominis: myiase "furonculeuse" d'Amerique du Sud. Med Trop 2008; 68: 7-10.

[21] Marchi MJ, Pereira PA, Menezes RMT, Tubaki RM. New records of mosquitoes carrying Dermatobia hominis eggs in the state of Sao Paulo, southeastern Brazil. J AM Mosq Control Assoc 2012; 28: 116-8.

[22] Heath AC, Elliot DC, Dreadon RG. Gasterophilus intestinalis the horse bot-fly as a cause of cutaneous myiasis in man. N Z Med J 1968; 68:31-2.

[23] Calderon HP, Rojas EC, Apt BW, Castillo OD. Cutaneous myiasis due to Cochliomyia hominivorax associated with seborrheic dermatidis. Rev Med Chil 2017; 45: 250- 4.

[24] Vijay Kumar GS, Sowmya GS, Shivananda S. Chrysomya bezziana oral myiasis. J Glob Infect Dis 2011; 3: 393-6.

[25] Cozzani E, Cioni M, Gariazzo L, et al. Furuncular myiasis dues to Wohlfahrtia magnifica. Eur J Dermatol 2017; 27: 402-3.

[26] Degeilh B, Beaucournu JC. A propos d'un agent de myiase fréquent en France méridionale Wohlfahrtia magnifica (Schiner,1862) (Diptera, Sarcophagidae). Bull Soc Entom France 2008 ; $113: 413-9$.

[27] Delhaes L, Bourel B, Pinatel F, et al. Myiase nasale humaine à Oestrus ovis. Parasite, 2001; $8: 289-96$.

[28] Anane S, BEN Hssine L. La myiase conjonctivale humaine à Oestrus ovis dans le sud tunisien. Bull Soc Path Exo 2010; 103 :299-304.

[29] Drummond RO, George JE, Kunz SE. Control of Arthropod Pests of livestock: a review of technologie. USA: CRC Press; 1988. 245pp. 
[30] Boulard C, Alvinerie M, Argenté G, et al. A successful sustaitable and low cost control programme for bovine hypodermosis in France. Vet Parasitol 2008; 158: 1-10.

[31] Bruel H, Guiguen C, Chevrier S, et al. Hypodermose humaine. Med Mal Infect 1995 ; 25 : 965-9.

[32] Doby JM, Quilici M, Mary C, Deunff J. Valeur comparée de l'immunoélectrophorèse et de I'ELISA dans le diagnostic sérologique de l'hypodermose humaine. Pathol Biol 1987 ; 35 : 381 5.

[33] Desoubeaux G, Gaillard J, Borée -Moreau D, et al. Gastrointestinal symptoms resembling ulcerative proctitis caused by larvae of the drone fly Eristalis tenax. Pathog Glob Health 2014; 108: 158- 63.

[34] Anofel. Myiases. In Parasitologie et mycologie médicales: guide des analyses et pratiques diagnostiques. Paris : Elsevier 2018. p377-81.

[35] Greco JB, Sacramento E, Tavares -Neto J. Chronic ulcers and myasis as ports of entry for Clostridium tetani. Braz J Infect Dis 2001;5:319-23.

[36] Clyti E, Couppie P, Cazanave C, et al. Traitement des myiases dues à Cochliomyia hominivorax par application locale d'ivermectine. Bull Soc Path Exot 2003 ; 96 : 410-11.

[37] Navy JE. Screwworm control and eradication in the southerm United States of America. World Animal Review 1991; www.fao.org.

[38] Lindquist DA, Abusow AM, Hall MJR. The new world screwworm fly in Libya : a review of introduction and eradication. Med Vet Entomol 1992; 6 : 2-8.

[39] Guiguen C. Arthropodes ectoparasites ou nuisants et autres invertébrés in Médecine tropicale. Paris : Lavoisier 2012. p 418-31.

[40] Cuisance D, Barré N, De Deken R. Ectoparasites des animaux: méthodes de lutte écologique, biologique, génétique et mécanique. Rev Sci Tech Off Int Epiz $1994 ; 13$ : 1305-56.

\section{Légende des figures}

Fig. 1: Cycle évotutif d'une mouche (larve, puparium et imago) : ici C. antropophaga (D. Basset- Montpellier) 
Fig. 2 : Eristalis tenax, la larve à queue de rat (e.anofel - Rennes)

Fig.3: Myiase furonculeuse : à gauche les 2 points noirs correspondent aux stigmates respiratoires; à droite sortie spontanée de la larve (e anofel - Rennes)

Fig.4: Lésions furonculeuses multiples à C. anthropophaga (e.anofel- Rennes)

Fig.5: Ver Macaque (larve de Dermatobia hominis). Noter les épines cuticulaires bien développées (e.anofel - )

Fig.6: Myiase d'une plaie anfractueuse du pavillon de l'oreille et de la joue (A. Lénot, Lannester).

Fig.7: Larve stade I d'Oestrus ovis extraite d'une conjonctive (D. Basset- Montpellier)

Fig.8: CEdème migrant de la face due à Hypoderma bovis (Parasitologie - Angers)

Fig.9 : Ophalmomyiase due à Hypoderma bovis chez un enfant (e.anofel - Rennes)

Fig.10 : Extraction d'une larve à la curette mousse (Parasitologie - Rennes)

Fig.11 : Extraction manuelle d'une larve de myiase furonculeuse (e.anofel -)

Fig.12 : Larves de stade III - Stigmates respiratoires de : 12 a C. anthropophaga , 12b $D$. hominis, 12c O. ovis, $12 \mathrm{~d}$ Rhinoestrus spp, 12e M. domestica, $12 \mathrm{f} C$. erythrocephala, $12 \mathrm{~g} L$. sericata, $12 \mathrm{~h} C$. hominivorax, $12 i \mathrm{~W}$. magnifica, $12 \mathrm{j}$ C. bezzania et sclérites buccaux en vue latérale de $12 \mathrm{k}$ C. erythrocephala et de $12 \mathrm{l}$ L. sericata (Parasitologie - Rennes)

Tableau 1- Principales myiases rencontrées en pathologie humaine

\begin{tabular}{|c|c|c|c|}
\hline Entités cliniques & $\begin{array}{c}\text { Type de } \\
\text { parasitisme }\end{array}$ & $\begin{array}{c}\text { Espèces } \\
\text { géographique }\end{array}$ \\
\hline épicutanée & obligatoire & Auchmeromyia senegalensis & Afrique noire \\
\hline
\end{tabular}




\begin{tabular}{|c|c|c|c|}
\hline (hématophage) & & & \\
\hline des plis & opportuniste & $\begin{array}{c}\text { Musca domestica } \\
\text { Calliphora erythrocephala }\end{array}$ & cosmopolite \\
\hline des plaies & $\begin{array}{c}\text { opportuniste } \\
\text { obligatoire }\end{array}$ & $\begin{array}{c}\text { Musca, Calliphora, Lucilia } \\
\text { Cochliomyia hominivorax } \\
\text { Wohlfahrtia magnifica } \\
\text { Chrysomya bezziana }\end{array}$ & $\begin{array}{l}\text { cosmopolite } \\
\text { Amérique } \\
\text { Europe } \\
\text { Afrique, Asie }\end{array}$ \\
\hline furonculeuse & obligatoire & $\begin{array}{c}\text { Hypoderma bovis } \\
\text { Cordylobia anthropophaga } \\
\text { Dermatobia hominis }\end{array}$ & $\begin{array}{l}\text { cosmopolite } \\
\text { Afrique noire } \\
\text { Amérique latin }\end{array}$ \\
\hline conjonctivale & obligatoire & Oestrus ovis & $\begin{array}{c}\text { bassin } \\
\text { méditerranéen }\end{array}$ \\
\hline $\begin{array}{l}\text { sous cutanée } \\
\text { rampante }\end{array}$ & obligatoire & Gasterophilus intestinalis & cosmopolite \\
\hline $\begin{array}{c}\text { cavitaire : } \\
\text {-vagin } \\
\text {-intestin } \\
\text {-conduit auditif } \\
\text {-sinus, nez }\end{array}$ & $\begin{array}{l}\text { opportuniste } \\
\text { opportuniste } \\
\text { opportuniste } \\
\text { obligatoire }\end{array}$ & $\begin{array}{c}\text { Musca domestica } \\
\text { Eristalis tenax, Fannia } \\
\text { Musca, Calliphora, Lucilia } \\
\text { Oestrus ovis, Rhinoestrus } \\
\text { purpureus }\end{array}$ & $\begin{array}{r}\text { cosmopolite } \\
\text { Europe } \\
\text { cosmopolite } \\
\text { Europe (sud), Afri }\end{array}$ \\
\hline intraoculaire & obligatoire & Hypoderma bovis & cosmopolite \\
\hline S N central & obligatoire & Hypoderma bovis & cosmopolite \\
\hline
\end{tabular}

Tableau 2- Clé de détermination : approche diagnostic à partir des formes clinique d'après [34] ( figure 12)

1- Asticot vu accroché au patient pendant la nuit en Afrique noire : 


\section{Auchmeromyia senegalensis}

2- Asticot issu d'un pseudofuroncle

2a - chez un patient revenant d'Afrique de l'Ouest, séjour en zone urbanisée ou anthropique: Cordylobia anthropophaga; les épines cuticulaires couvrent tout le corps, les fentes des stigmates sont sinueuses et convergent vers le bouton (12a).

$2 b$ - chez un patient revenant d'Afrique de l'Ouest, séjour fréquent en zone forestière: Cordylobia rodhaini ; les épines cuticulaires couvrent tout le corps, les fentes des stigmates très sinueuses et convergent vers le bouton. Le péritrème à un grand diamètre.

2c - chez un patient revenant d'Amérique latine : Dermatobia hominis ; les épines cuticulaires sont en épines de rosier et forme une rangée par segment; les fentes des spiracles respiratoires sont rectilignes $(12 b)$.

$2 d$ - Chez un jeune patient $(<15 a n s)$ n'ayant pas quitté la France et ayant séjourné en zone rurale : Hypoderma bovis ; la larve translucide de stade1 mesure $1 \mathrm{~cm}$ environ.

3 - Asticot issu des fausses nasales ou vu sur la conjonctive de l'œil

$3 a$ - les orifices stigmatiques sont dispersés et ponctiformes à l'intérieur du péritrème, le bouton est inséré au centre du stigmate : Oestrus ovis (12c).

$3 b$ - les orifices stigmatiques sont dispersés et ponctiformes à l'intérieur du péritrème le bouton est plus ou moins invaginé en bord latéro interne du stigmate: Rhinoestrus purpureus (12d).

4 - Asticot trouvé dans les selles

$4 a$ - stigmates portés par un long filament postérieur «larve à queue de rat » : Eristalis tenax.

4b - stigmates accolés au corps et cuticule hérissée latéralement de longs filaments en feuille de fougère : Fannia spp

5 - Asticot issu des plis, des plaies, du conduit auditif, du vagin,...

$5 a$ - fentes stigmatiques sinueuses se suivant bout à bout : Musca domestica (12e) 
$5 b$ - fentes rectilignes convergentes vers le bouton du péritrème: 6

6 - bouton enchâssé dans le péritrème : $7(12 f, 12 \mathrm{~g})$

-bouton non enchâssé dans le péritrème : 8

7a - présence d'un minuscule sclérite buccal au niveau de l'apex du crochet buccal : Calliphora erythrocephala (12k).

7b - absence d'un minuscule sclérite buccal au niveau de l'apex du crochet buccal : Lucilia sericata (12l).

$8 \mathrm{a}$ - péritrème interrompu et bouton peu sclérifié : Cochliomyia hominivorax (12h)

$8 \mathrm{~b}$ - péritrème largement interrompu, bouton absent ou peu visible 9

9a - péritrème sans saillie sclérotisée vers l'intérieur : Wohlfahrtia magnifica (12i)

9b - péritrène avec saillie sclérotisée vers l'intérieur : Chrysomya spp (12j) 

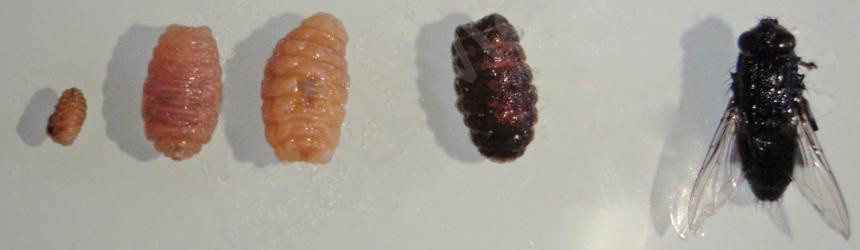


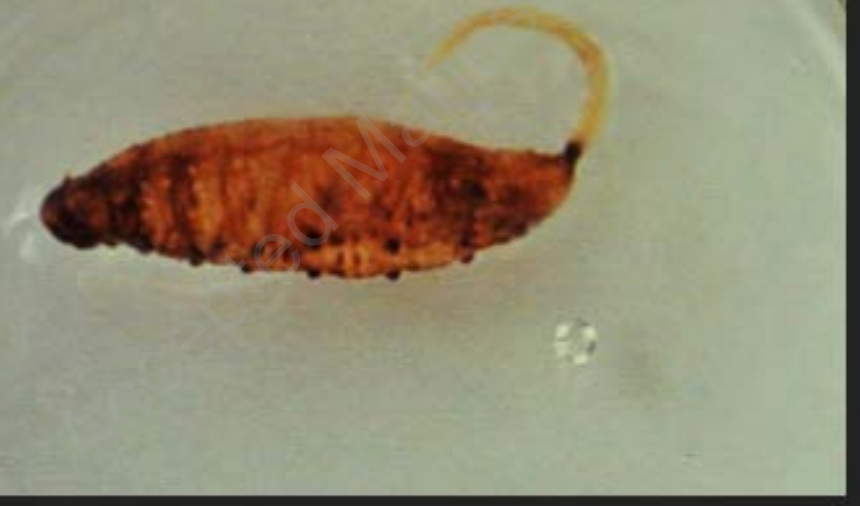





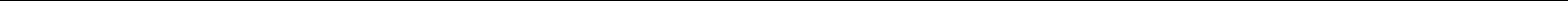



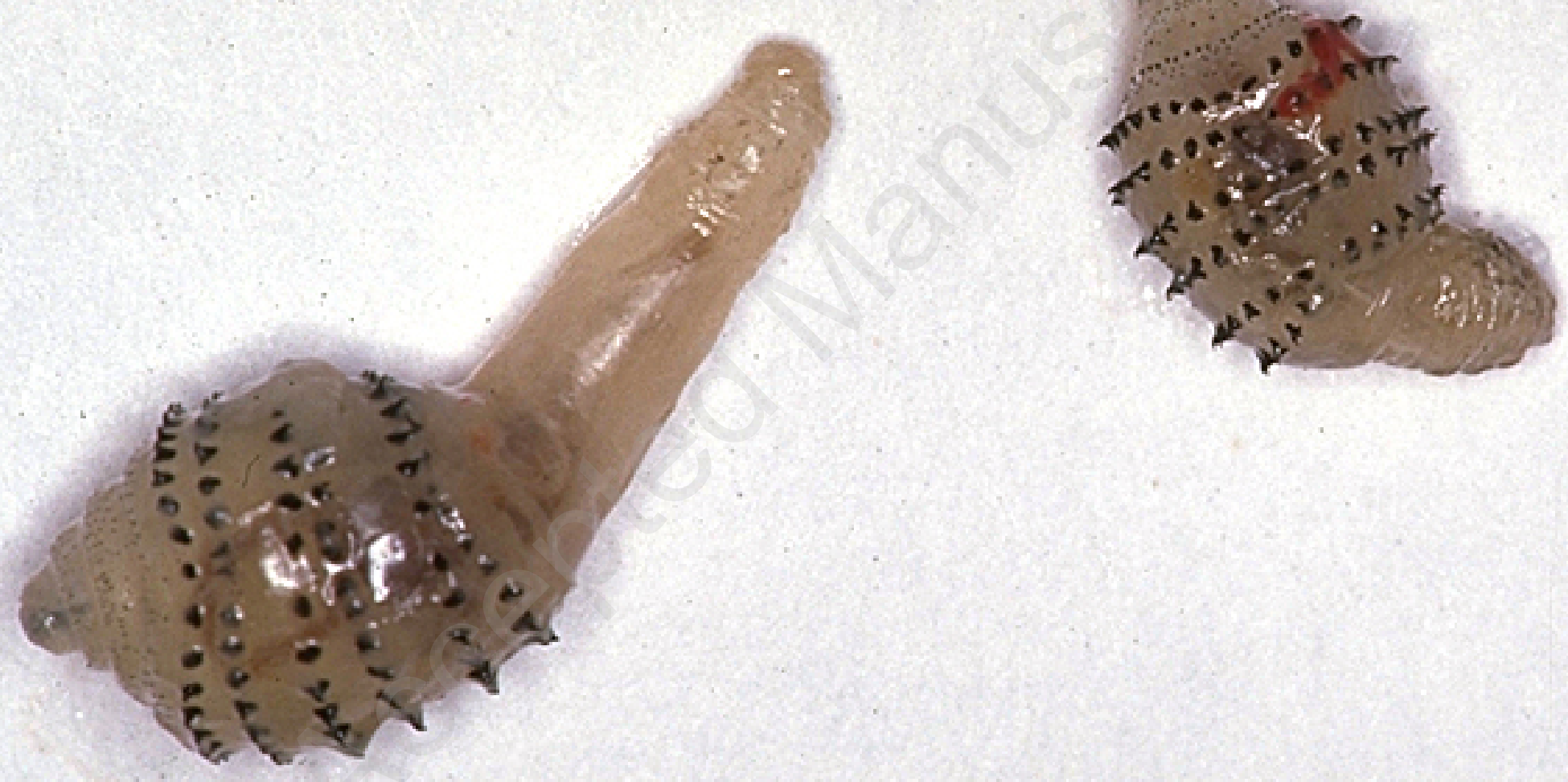

\section{ANOFEL}


" al

24A

III

79.

क्ष
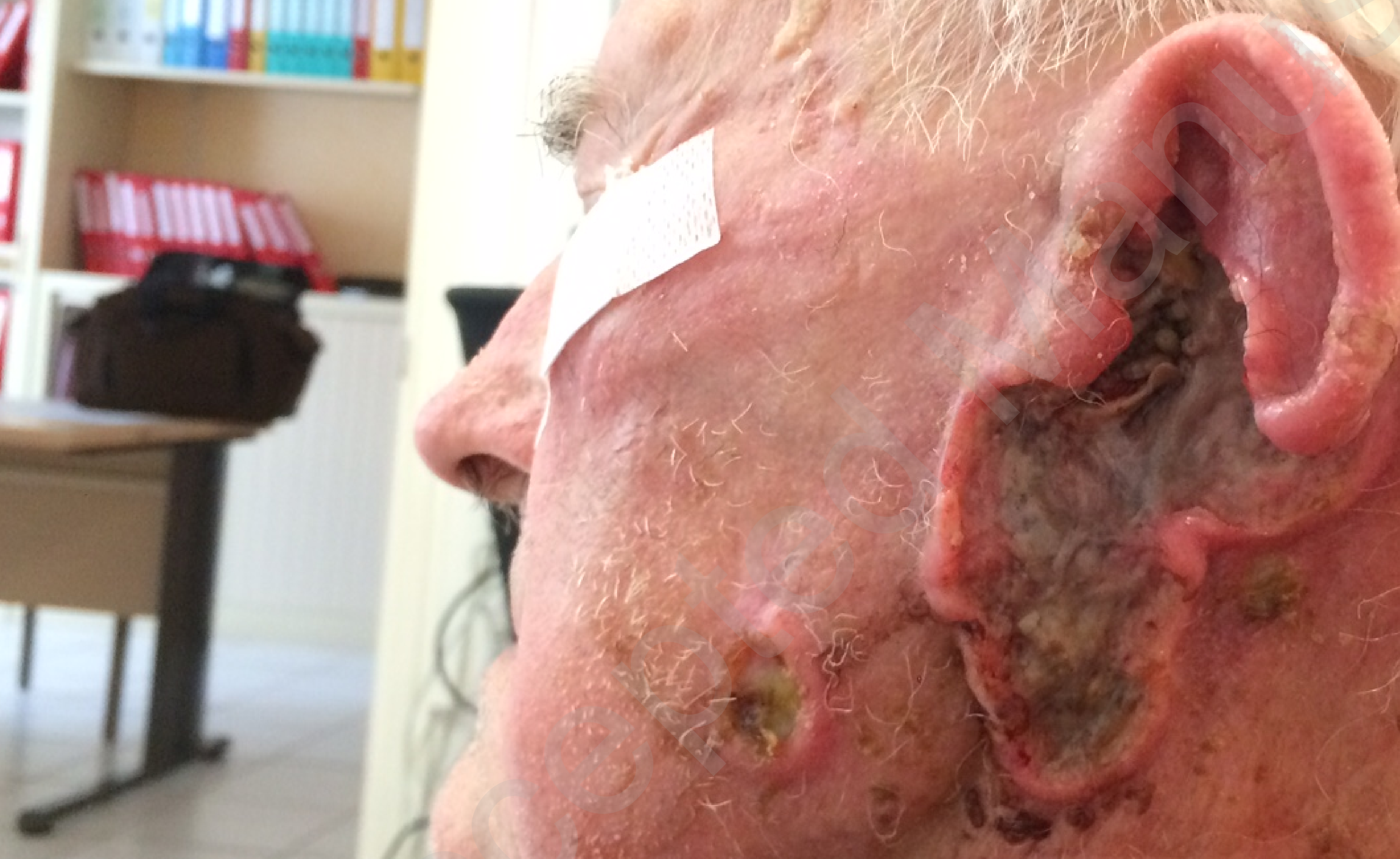


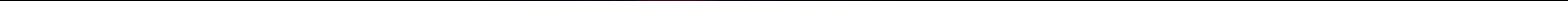





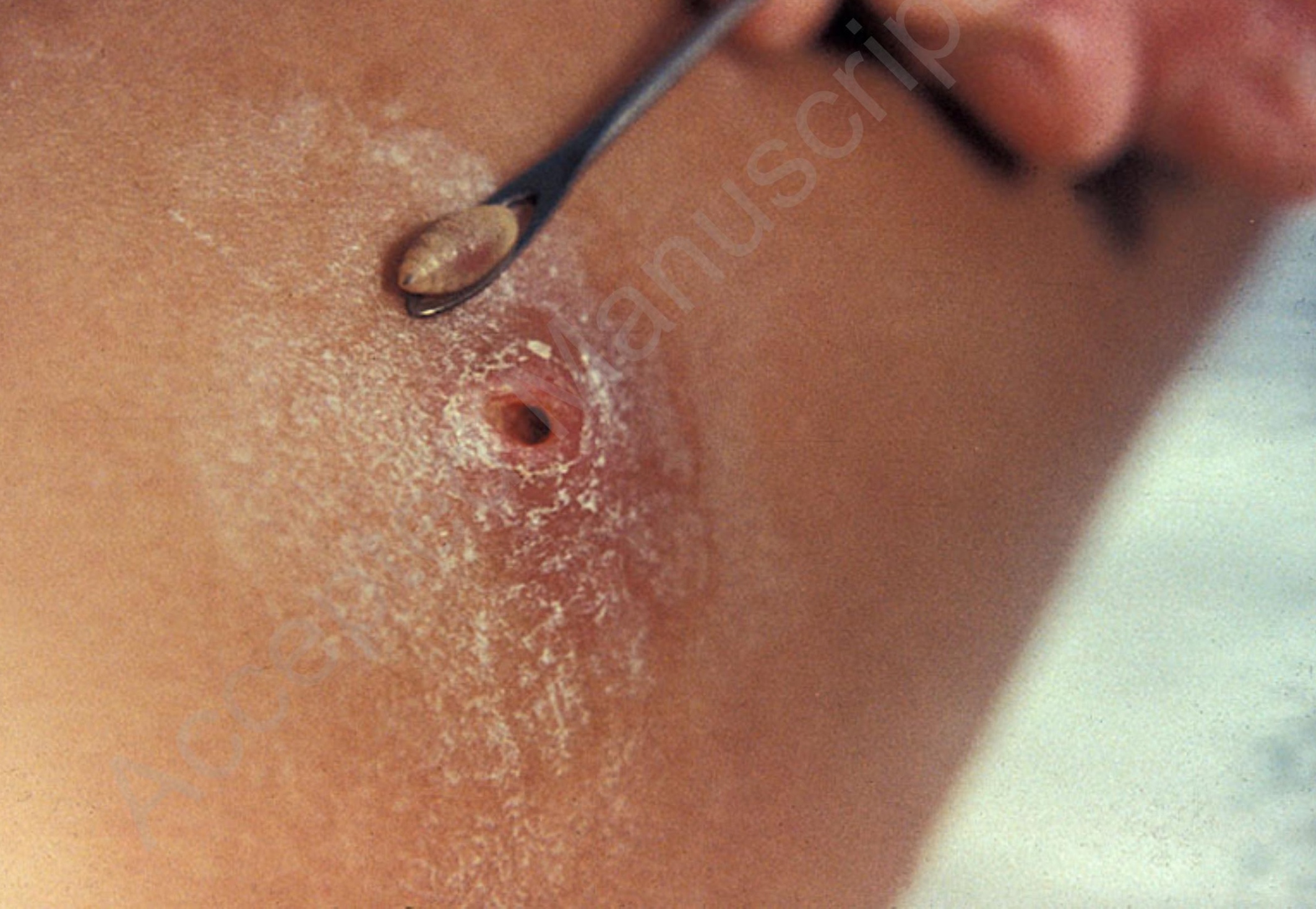




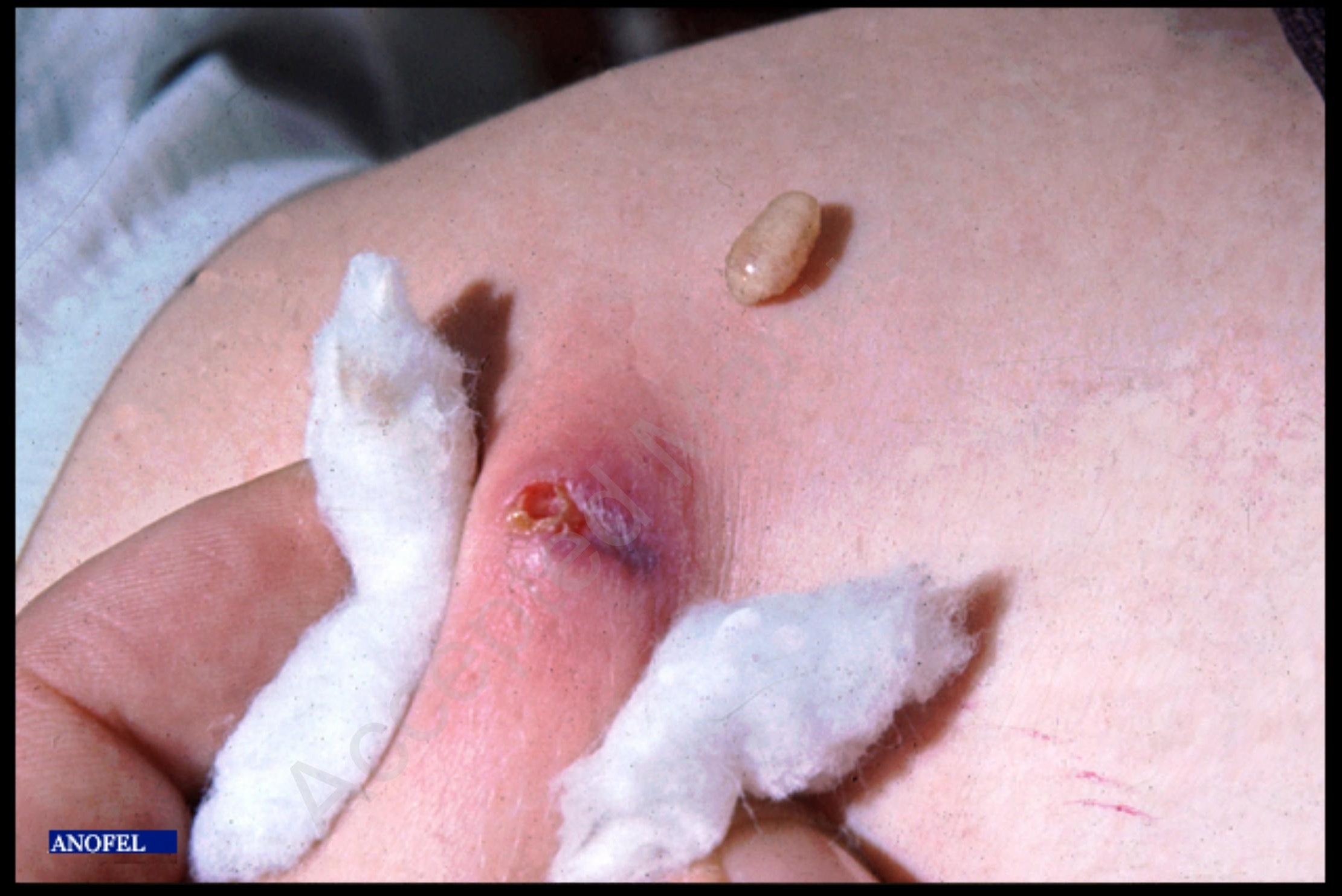

